\title{
ON THE COHOMOLOGY GROUPS OF A MANIFOLD WITH A NONINTEGRABLE SUBBUNDLE
}

\author{
HARUO KITAHARA AND SHINSUKE YOROZU
}

\begin{abstract}
We define the cohomology groups $H^{u}\left(M, \mathscr{D}^{s, t}\right)$ of a manifold $M$ with a nonintegrable subbundle $E$, and we give the condition for the existence of a bundle-like metric with respect to $E$.
\end{abstract}

1. Introduction. N. Abe [1], J. L. Heitsch [3] and I. Vaisman [6] studied some cohomology groups of a manifold with a foliation ("integrable case"). In this note, we generalize their results to the nonintegrable case, that is, we define the cohomology groups $H^{u}\left(M, \mathcal{O}^{s, t}\right)$ of a manifold $M$ with a nonintegrable subbundle $E$. In the case that $M$ is a riemannian manifold, by its cohomology groups, we give the condition for the existence of a bundle-like metric with respect to $E$.

2. Preliminaries. We shall be in $C^{\infty}$-category. Let $M$ be an $n$-dimensional paracompact manifold with tangent bundle $T M$. Let $E$ be a subbundle of $T M$ with the constant fibre dimension $n-p(0<p<n)$. We assume that $E$ is not integrable. $\Gamma(\cdot)$ denotes the functor associating to a bundle its vector space of sections, and [,] the bracket operator on $\Gamma(T M)$. Let $C(E)$ be the "Cauchy characteristic subbundle" of $E$, i.e. the fibre $C_{x}(E)$ over $x \in M$ of $C(E)$ consists of $X_{x} \in E_{x}$ (= the fibre over $x$ of $E$ ) such that $[X, Y]_{x} \in E_{x}$ for any $Y_{x} \in E_{x}, X, Y \in \Gamma(E)$, and, for all $x \in M, \operatorname{dim} C_{x}(E)$ is assumed to be constant. Then $C(E)$ is an integrable subbundle of $E$ (naturally, of $T M$ ). We assume that the fibre dimension of $C(E)$ is $n-q(0<p<q<n)$. We set

$$
Q=T M / E, \quad E^{\prime}=E / C(E),
$$

and, by a suitable riemannian metric on $T M$, we have isomorphisms

$$
\begin{gathered}
\Gamma(T M)=\Gamma(Q) \oplus \Gamma(E), \quad \Gamma(T M)=\Gamma(T M / C(E)) \oplus \Gamma(C(E)), \\
\Gamma(T M)=\Gamma(Q) \oplus \Gamma\left(E^{\prime}\right) \oplus \Gamma(C(E)) .
\end{gathered}
$$

3. $(s, t, u)$-forms and cohomology groups $\left.H^{u}(M, \mathcal{O})^{s, t}\right)$. Let $A^{r}$ be the space of all $r$-forms on $M$ and $d$ the exterior derivative.

Definition. An $r$-form $\omega \in A^{r}$ is a $(s, t, u)$-form, if

(i) $s+t+u=r$, and

Received by the editors September 3, 1975 and, in revised form, December 8, 1975.

AMS (MOS) subject classifications (1970). Primary 14F05, 55B30, 57D30.

Key words and phrases. Cohomology groups, Cauchy characteristic subbundle, sheaf, generalized Bott connection, bundle-like metric with respect to $E$. 
(ii) $\omega\left(X_{1}, \ldots, X_{r}\right)=0$ except for $s$ arguments $X_{i}$ 's in $\Gamma(Q)$, $t$ arguments $X_{i}$ 's in $\Gamma\left(E^{\prime}\right)$ and $u$ arguments $X_{i}^{\prime}$ s in $\Gamma(C(E))$.

Let $A^{s, t, u}$ be the space of all $(s, t, u)$-forms on $M$, and we have a decomposition

$$
A^{r}=\sum_{s+t+u=r} A^{s, t, u}
$$

By the definition of $C(E)$, we have

(3) $[\Gamma(C(E)), \Gamma(E)] \subset \Gamma(E), \quad[\Gamma(C(E)), \Gamma(C(E))] \subset \Gamma(C(E))$.

From this, we have that the partial derivative

$$
\hat{\partial}: A^{s, t, u} \rightarrow A^{s, t, u+1}
$$

induced by the exterior derivative satisfies $(\hat{\partial})^{2}=0$. Let $c^{s, t, u}$ be the sheaf of germs of $(s, t, u)$-forms. Then each $\left(^{\prime s, t, u} \text { is a fine sheaf. Let }{ }^{\prime}\right)^{s, t}$ be the sheaf defined by $\left.0^{s}\right)^{s, t}=\mathbb{C}^{s, t, 0} \cap \operatorname{ker}(\hat{\partial})$.

REMARK. ${ }^{0}{ }^{0,0}$ denotes the sheaf of germs of functions which are constants on the leaves of $C(E)$.

THEOREM 1. There exists a fine resolution of the sheaf

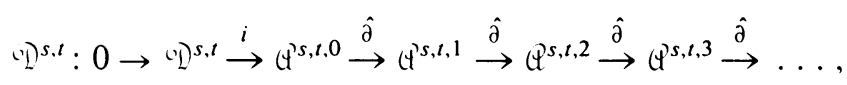

where $i$ denotes the natural inclusion.

Proof. Since we can obtain the Poincare lemma for the operator $\hat{\partial}$ and the $(s, t, u)$-form on the open unit ball in euclidean $n$-space (cf. [6]), we easily prove the assertion of the theorem.

Let $H^{u}\left(M, O D^{s, t}\right)$ be the cohomology groups of $M$ with coefficients in the sheaf $0_{2} s, t$. Then we have

THEOREM 2. There exist isomorphisms

$$
\begin{gathered}
H^{0}\left(M, \text { o }\left.\right|^{s, t}\right) \cong A^{s, t, 0} \cap \operatorname{ker}(\hat{\partial}), \\
H^{u}\left(M, \mathcal{D Q D}^{s, t}\right) \cong A^{s, t, u} \cap \operatorname{ker}(\hat{\partial}) / \hat{\partial} A^{s, t, u-1} \text { for } u \geqslant 1 .
\end{gathered}
$$

Corollary 3. $H^{u}\left(M, \mathcal{O}^{s, t}\right) \cong\{0\}$ for $s>p$ or $t>q-p$ or $u>n-q$.

4. Generalized Bott connection and cohomology groups $H^{u}(M$, 이 $s, t(Q))$. Let $\pi: T M \rightarrow Q=T M / E$ be the canonical projection. We define a map

$$
\hat{\nabla}: \Gamma(C(E)) \times \Gamma(Q) \rightarrow \Gamma(Q)
$$

by

$$
\hat{\nabla}_{X}(S)=\pi_{*}([X, \tilde{S}])
$$

for $\forall X \in \Gamma(C(E)), \forall S \in \Gamma(Q)$ and $\tilde{S} \in \Gamma(T M)$ such that $\pi_{*}(\tilde{S})=S$. From (3), this is well defined. Let $\nabla^{\prime}$ be any connection on $Q$. For $X \in$ $\Gamma(T M)$, from (2), we can write $X=X_{1}+X_{2}, X_{1} \in \Gamma(C(E)), X_{2} \in$ $\Gamma(T M / C(E))$. Thus we define a map

$$
\nabla: \Gamma(T M) \times \Gamma(Q) \rightarrow \Gamma(Q)
$$


by

$$
\nabla_{X}(S)=\hat{\nabla}_{X_{1}}(S)+\nabla_{X_{2}}(S) .
$$

Then $\nabla$ is a connection on $Q$, and is called a generalized Bott connection (cf. [2], [4]).

Let $A^{s, t, u}(Q)$ be the space of all $Q$-valued $(s, t, u)$-forms on $M$ and $\mathbb{Q}^{s, t, u}(Q)$ the corresponding sheaf. $\hat{\partial}$ operating on $A^{s, t, u}(Q)$ is given by $\nabla$. Then, as above, we have a fine resolution of the sheaf $(1)^{s, t}(Q)=Q^{s, t, 0}(Q) \cap$ $\operatorname{ker}(\hat{\partial})$. Thus we have

THEOREM 4. There exist isomorphisms:

$$
\begin{gathered}
H^{0}\left(M, \mathscr{Q}^{s, t}(Q)\right) \cong A^{s, t, 0}(Q) \cap \operatorname{ker}(\hat{\partial}), \\
H^{u}\left(M, \mathscr{D}^{s, t}(Q)\right) \cong A^{s, t, u}(Q) \cap \operatorname{ker}(\hat{\partial}) / \hat{\partial} A^{s, t, u-1}(Q) \quad \text { for } u \geqslant 1 .
\end{gathered}
$$

REMARK. If $E$ is integrable, the same results are given by $\mathbf{J}$. L. Heitsch [3].

5. Bundle-like metric with respect to $E$. Let $M$ be an $n$-dimensional riemannian manifold with the metric $g$, and $E^{\perp}$ the orthogonal complement of $E$ in $T M$. Let $\hat{\nabla}: \Gamma(C(E)) \times \Gamma\left(E^{\perp}\right) \rightarrow \Gamma\left(E^{\perp}\right)$ be a map defined by $\hat{\nabla}_{X}(S)=\pi_{*}([X, S])\left(\pi: T M \rightarrow E^{\perp}\right.$ the canonical projection), we define a connection $\nabla$ on $E^{\perp}$ as in (5).

Definition. The riemannian metric $g$ is a bundle-like metric with respect to $E$, if $\left(\nabla_{X} g\right)\left(S_{1}, S_{2}\right)=0$ for $\forall X \in \Gamma(C(E)), \forall S_{1}, \forall S_{2} \in \Gamma\left(E^{\perp}\right)$.

In the following, we assume that the fibre dimension of $E$ is $n-1$. Let $\left\{e_{A}\right\}$ be an orthonormal frame such that $e_{1} \in \Gamma\left(E^{\perp}\right)$ and $e_{a} \in \Gamma(E)$, and $\left\{\omega^{A}\right\}$ its dual $(1 \leqslant A \leqslant n, 2 \leqslant a \leqslant n)$. We assume that $\omega^{1}$ is a global form (if necessary, we assume that $E$ is transversally orientable).

LemMA 5. $g$ is a bundle-like metric with respect to $E$ if and only if $\nabla_{X}\left(e_{1}\right)=0$ for $\forall X \in \Gamma(C(E))$.

Proof. For $\forall S_{1}=\xi \cdot e_{1}, \forall S_{2}=\eta \cdot e_{1} \in \Gamma\left(E^{\perp}\right)(\xi, \eta$ : functions),

$$
\begin{aligned}
\left(\nabla_{X} g\right)\left(S_{1}, S_{2}\right)= & X\left(g\left(S_{1}, S_{2}\right)\right)-g\left(\nabla_{X}\left(S_{1}\right), S_{2}\right)-g\left(S_{1}, \nabla_{X}\left(S_{2}\right)\right) \\
= & X(\xi \cdot \eta)-\eta \cdot X(\xi)-(\xi \cdot \eta) g\left(\nabla_{X}\left(e_{1}\right), e_{1}\right) \\
& -\xi \cdot X(\eta)-(\xi \cdot \eta) g\left(e_{1}, \nabla_{X}\left(e_{1}\right)\right) \\
= & -2(\xi \cdot \eta) g\left(e_{1}, \nabla_{X}\left(e_{1}\right)\right) .
\end{aligned}
$$

Thus we have the assertion of the lemma.

By the above metric $g$, we have an isomorphism $\Gamma(Q) \cong \Gamma\left(E^{\perp}\right)$, and we can identify the connections $\nabla$ on $Q$ and on $E^{\perp}$.

LeMma 6. $\hat{\partial} \omega^{1}=0$ if and only if $\nabla_{X}\left(e_{1}\right)=0$ for $\forall X \in \Gamma(C(E))$.

PROoF. For $\forall S=\xi \cdot e_{1}$ ( $\xi$ : function), 


$$
\begin{aligned}
\hat{\partial} \omega^{1}(S, X) & =-X\left(\omega^{1}(S)\right)-\omega^{1}([S, X])=-X(\xi)+\omega^{1}\left(\nabla_{X}(S)\right) \\
& =-X(\xi)+X(\xi)+\xi \cdot \omega^{1}\left(\nabla_{X}\left(e_{1}\right)\right) \\
& =\xi \cdot \omega^{1}\left(\nabla_{X}\left(e_{1}\right)\right) .
\end{aligned}
$$

Thus we have the assertion of the lemma.

From the above lemmas, we have

THEOREM 7. Let $M$ be an $n$-dimensional riemannian manifold with the metric $g$ and $E$ a nonintegrable, transversally orientable subbundle of TM of fibre dimension $n-1$. If $H^{0}\left(M, \mathcal{O}^{1,0}\right) \cong A^{1,0,0}$, then $g$ is a bundle-like metric with respect to $E$. Conversely, if $g$ is a bundle-like metric with respect to $E$, then $H^{0}\left(M, \mathscr{D}^{1,0}\right) \neq\{0\}$.

Proof. A nonzero 1 -form $\omega^{1}$ is a $(1,0,0)$-form on $M$. If $H^{0}\left(M, \mathcal{o l}^{1,0}\right) \cong$ $A^{1,0,0}$, then $A^{1,0,0} \cong A^{1,0,0} \cap \operatorname{ker}(\hat{\partial})$ and we have $\hat{\partial} \omega^{1}=0$. By Lemmas 5 and $6, g$ is a bundle-like metric with respect to $E$. Conversely, if $g$ is a bundle-like metric with respect to $E$, by Lemmas 5 and 6 , we have $\hat{\partial} \omega^{1}=0$ and $\omega^{1}$ is a nonzero $(1,0,0)$-form on $M$. Thus we have $\left.H^{0}(M, \text { o })^{1,0}\right) \neq\{0\}$.

REMARK. In the case that $E$ is integrable, $M$ is compact and $g$ is a bundle-like metric, then $H^{1}(M, R) \neq\{0\}$ (cf. R. Sacksteder [5]).

\section{REFERENCES}

1. N. Abe, On cohomology and characteristic classes of a foliated manifold (preprint).

2. R. Bott, Lectures on characteristic classes and foliations, Lecture Notes in Math., vol. 279, Springer-Verlag, Berlin and New York, 1972, pp. 1-80.

3. J. L. Heitsch, A cohomology for foliated manifolds, Bull. Amer. Math. Soc. 79 (1973), 1283-1285.

4. H. Kitahara and S. Yorozu, A generalized Godbillon-Vey invariant for a subbundle which is not integrable (preprint).

5. R. Sacksteder, Foliations and pseudogroups, Amer. J. Math. 87 (1965), 79-102. MR 30 \#4268.

6. I. Vaisman, Cohomology and differentiable forms, Dekker, New York, 1973. MR 49 \#6095.

Department of Mathematics, College of Liberal Arts, Kanazawa University, KANAZAWA, JAPAN 